\title{
«Sono una madre single. Non sento di poter essere competitiva quanto altri»: esperienze di personale precario nelle università del Regno Unito
}

Thomas Allmer, University of Stirling, Department of Communications, Media and Culture thomas.allmer@stir.ac.uk

Translated by Gloria Dagnino

From the English paper: Allmer, T. (2018). "I am a single mum. I don't feel like I can be as competitive as other people": Experiences of precariously employed staff at UK universities. Work Organisation, Labour \& Globalisation, 12(1), 62-77.

\begin{abstract}
Universities play a central role in informational capitalism. However, higher education institutions have undergone economic, political and cultural transformations leading to competition, market orientation and new management forms. These changes have effects on many levels, including the working conditions and practices of individuals involved in the information gaining process. This article aims to find out how the existing working conditions and practices at universities form the meanings, identities and experiences of individuals by focusing on precariously employed academics. I address this question based on a theoretical analysis and qualitative interviews with casualised academic staff.
\end{abstract}

\section{Keywords}

academic labour, universities, information work, precarious work, casualisation, informational capitalism

\begin{abstract}
Le università giocano un ruolo centrale nel capitalismo informazionale. Tuttavia, le istituzioni di istruzione superiore hanno subito trasformazioni economiche, politiche e culturali che hanno generato concorrenza, orientamento al mercato e nuove forme di management. Questi cambiamenti producono effetti a molti livelli, tra cui le condizioni di lavoro e le pratiche degli individui che partecipano al processo di raccolta delle informazioni. Questo articolo intende scoprire come le condizioni di lavoro e le pratiche in atto nelle università diano forma ai significati, alle identità e alle esperienze degli individui, concentrandosi sul personale precario. Affronto questa questione sulla base di un'analisi teorica e di interviste qualitative con personale accademico impiegato in modo precario.
\end{abstract}

\section{Parole chiave}

Lavoro accademico, università, lavoro dell'informazione, lavoro precario, precarizzazione, capitalismo informazionale

\section{Note}

Invited paper for the Thematic Section on ECREA 2018 devoted to the $7^{\text {th }}$ European Communication Conference of the European Communication and Research Association (ECREA), held in Lugano from October $31^{\text {st }}$ to November $3^{\text {rd }}, 2018$. 


\section{Introduzione}

Le trasformazioni economiche e politiche che hanno interessato le università negli ultimi decenni hanno suscitato critiche da più parti. Ciò emerge anche dal volume crescente di letteratura accademica che indaga questi cambiamenti nel contesto del neoliberismo e del crescente intreccio tra istituzioni private e pubbliche. All'interno delle università, si è accuratamente incoraggiato e prodotto un nuovo spirito imprenditoriale e manageriale che ha portato all'applicazione delle regole di mercato e della concorrenza (Deem, Hyllyard e Reed, 2007). È stato detto che, oggigiorno, le istituzioni di istruzione mirino a soddisfare le esigenze del mercato mentre il carattere pubblico dell'istruzione tende a scomparire (Peters, 2003). Gli studiosi appartenenti alla scuola critica parlano di «capitalismo accademico» (Slaughter e Leslie, 1999), di «università su modello aziendale» (Giroux, 2002) e di «Uber.edu» (Hall, 2016). Queste trasformazioni strutturali hanno avuto diverse conseguenze sulle condizioni di lavoro, le pratiche e le relazioni delle persone, tra cui, per citarne solo alcune, l'intensificazione e l'estensione del lavoro, il confine sempre più sfumato tra lavoro e tempo libero, la tendenza all'impiego occasionale, la precarietà, l'auto-sfruttamento e l'auto-promozione. Il modo in cui queste condizioni vengono esperite da soggetti diversi è una questione tuttora aperta al dibattito. Mentre le esperienze di lavoro in altri settori come le industrie culturali e creative sono ben documentate, vi è ancora poca comprensione delle soggettività dei lavoratori nel mondo accademico, così come manca un'analisi di come le condizioni esistenti vengano vissute dagli accademici (Gill, 2014, 12-13).

Questo articolo cerca di trovare risposte alla seguente domanda: in che modo le condizioni e le pratiche di lavoro esistenti nelle università vengono percepite e vissute dagli accademici che sono impiegati in modo precario?

Affronto questa domanda sulla base di un'analisi teorica e di interviste qualitative con il personale accademico precario im- piegato presso alcune istituzioni di istruzione superiore scozzesi. In particolare, la prossima sezione delinea alcune basi teoriche che riguardano lo studio del lavoro accademico. La sezione successiva presenta la metodologia della ricerca empirica che è stata condotta. Successivamente, alcuni risultati dello studio vengono presentati e discussi in relazione ai temi della precarietà del lavoro, del carico di lavoro, del management, dei meccanismi di controllo, della salute mentale e fisica e del genere. L'articolo si conclude con un riassunto e con la discussione di alcune implicazioni ulteriori di questo studio.

\section{Basi teoriche}

In questa sezione, delineerò alcune basi teoriche riguardanti lo studio del lavoro accademico: in particolare, insicurezza occupazionale, carico di lavoro, management, meccanismi di controllo, salute mentale e fisica e infine genere. Queste sono alcune delle importanti dimensioni che danno forma alle condizioni di lavoro negli istituti di istruzione superiore. Per un modello sistematico delle condizioni di lavoro nelle università si veda Allmer (2017).

\section{Insicurezza occupazionale}

L'impiego nel settore dell'istruzione superiore è caratterizzato da una tendenza alla precarizzazione e al ricorso a contratti a tempo. Secondo la Higher Education Statistics Agency (2016), nell'anno accademico 2014/2015, 128300 persone occupate nelle università del Regno Unito avevano un contratto permanente o a tempo indeterminato e 70035 avevano un contratto a tempo determinato. A ciò si aggiungevano, nello stesso anno, 75560 persone impiegate nel settore accademico con contratti atipici. Se si sommano i lavoratori con contratto a tempo determinato e i lavoratori con contratti atipici risulta che la maggioranza $(53.2 \%)$ del personale accademico del Regno Unito lavora nell'istruzione superiore su base temporanea. La precarizzazione permette alle università 
di mettere alla prova la performance degli accademici, rafforza la «selezione darwiniana», riduce i costi del lavoro e dà la possibilità di rispondere velocemente ai cambiamenti del mercato dell'istruzione per fare fronte ai picchi di crescita e calo della domanda (Bryson e Barnes, 2000, 193). La quantità di personale necessaria dipende anche dal successo che un'università ha in termini di marketing e di capacità di attirare studenti per il successivo anno accademico. Le università competono tra loro nel mercato per potenziali nuovi studenti. La precarizzazione del personale accademico può quindi essere considerata come uno dei risultati dell'applicazione, negli istituti di istruzione superiore, di regole vicine a quelle del mercato e neoliberali:

L'università non ha mai potuto essere certa della quantità o della redditività delle iscrizioni; ha dovuto rimanere sempre pronta ad agire, a stimolare le iscrizioni, a ridurre i costi, a continuare a crescere. La flessibilità permanente richiesta da questa situazione ha significato che il personale dovesse essere proletarizzato e stratificato in lavoratori temporanei a tempo parziale, docenti permanenti e ricercatori permanenti. (Shumar, 1995, 94)

Pratt (1997) ha sottolineato che l'impiego di personale a tempo parziale e a tempo determinato nelle università è diventata una strategia di management. A vent'anni da quando è stata effettuata questa analisi pionieristica, coloro che lavorano in un'università pre-1992 $19{ }^{1}$ sono ora generalmente assunti con contratti di sola ricerca, lavorano a tempo parziale, hanno meno di cinque anni di esperienza lavorativa, sono donne e hanno meno di 40 anni di età. Inoltre, il personale non bianco e non britannico ha maggiori probabilità di essere assunto con contratti temporanei (Higher Education Statistics Agency, 2016).

1 Le istituzioni di istruzione superiore nel Regno Unito si dividono tra quelle che hanno ricevuto lo status di università prima del 1992, per autorizzazione reale, e quelle che lo hanno ricevuto per effetto del Further and Higher Education Act del 1992 (nota della traduttrice).
I contratti a tempo determinato tendono ad avere un impatto sulla sicurezza economica e sulla capacità di controllo del dipendente, sulla sua esclusione dal dipartimento, sui rapporti con altri colleghi e sulla mancanza di opportunità di sviluppo professionale e di avanzamento di carriera (Bryson e Barnes, 2000, 217). Gulli $(2009,5)$ sottolinea che l'aumento del personale temporaneo è tipica del discorso neoliberale in quanto porta flessibilità all'università a scapito della sicurezza individuale, il ché può generare ansia, disturbi, stigmatizzazione e perdita di dignità. L'impiego di personale temporaneo è caratterizzato da una contraddizione tra inclusione ed esclusione. Il personale temporaneo è molto richiesto ed è incluso in termini economici, ma tende ad essere invisibile ed esposto, e quindi escluso, in termini sociali e politici. Tirelli (1999) ha quindi sottolineato che i contratti occasionali provocano segmentazioni del lavoro all'interno del personale accademico, con conseguente aumento delle gerarchie e del potenziale di conflitto. Le università neoliberali tendono a diminuire il numero di personale a tempo indeterminato, relativamente inserito e rispettato, e ad aumentare il numero di personale temporaneo, relativamente privo di potere. Ciò non significa, tuttavia, che solo il personale temporaneo subisca le conseguenze delle condizioni neoliberali, ma piuttosto che la precarizzazione interessa tutto il mondo accademico. Da un punto di vista sindacale, la precarietà porta anche cambiamenti politici che avvantaggiano la direzione e indeboliscono la forza lavoro accademica. «Di fronte a una massa incontrollabile di lavoro immateriale, la migliore strategia di un amministratore universitario - sostenuta da secoli di gerarchia accademica - è quella di assicurare che il personale accademico regolare e quello temporaneo rimangano divisi» (Dyer-Witheford, 2005, 78).

\section{Carico di lavoro}

Facendo un'analogia con l'idea della «fabbrica senza muri» (Dyer-Witheford, 1999, 80) elaborata dal marxismo autonomista, 
Gill (2010) sostiene che l'università neoliberale può essere considerata come una «accademia senza muri». Il marxismo autonomista sostiene che il capitale tende a sussumere l'intera società nel processo produttivo, così che la logica della fabbrica si estende a tutta la società (Wright, 2002: 37-38). La società funziona come una fase della produzione, dove il confine tra lavoro e tempo libero diventa sempre più confuso sia spazialmente che temporalmente (Gorz, 2010, 22). Così come la fabbrica sociale nel suo insieme è una fabbrica senza muri, le università neoliberali hanno intensificato il lavoro in termini di tempo e lo hanno esteso in termini di spazio, grazie alle tecnologie digitali. Gli accademici tendono ad avere confini fluidi tra lo spazio di lavoro e gli altri spazi della loro vita, e tra il tempo di lavoro e il tempo libero (Ross, 2000, 23). Le culture della connessione costante hanno trasformato l'università in una "fast-accademia». "Le tecnologie mobili sempre più accelerate si intrecciano senza soluzione di continuità con l'habitus psichico e con l'indole del soggetto accademico neoliberale: controllare, monitorare, scaricare contenuti dalla BL (British Library), come dalla spiaggia o dal letto, e cercare disperatamente di rimanere aggiornati e «sul pezzo»» (Gill, 2010, 237).

Questa affermazione teorica può essere sostenuta da dati empirici. La University and College Union ha condotto, tra i suoi membri nel Regno Unito, diverse indagini online sul carico di lavoro e sullo stress legato al lavoro (Court e Kinman, 2009; Kinman e Wray, 2013; University and College Union, 2014; University and College Union, 2016). Nel 2014 ( $n=6.439)$, il 79\% dei partecipanti si è dichiarato d'accordo o molto d'accordo sul fatto di trovare il proprio lavoro stressante. E il $53 \%$ ha indicato che il loro livello generale o medio di stress era alto o molto alto. Quasi la metà (48\%) ha risposto di vivere livelli inaccettabili di stress spesso o sempre (University and College Union, 2014, 1-2). Secondo l'indagine del 2016 ( $n=12.113)$, il personale accademico ha lavorato in media più dell'equivalente di un tempo pieno di 50 ore settimanali. Soprattutto tra gli accademici ad inizio carriera, prevaleva una cultura fatta di lunghe ore di lavoro (University and College Union, 2016, 18). Trai i fattori che contribuiscono allo stress nell'istruzione superiore vi erano, tra gli altri, la mancanza di tempo per il lavoro di ricerca, l'eccessivo carico di lavoro, i problemi nell'ottenere finanziamenti, la mancanza di opportunità di avanzamento di carriera e la precarietà del lavoro (Court e Kinman, 2009, 61). Gli accademici lavorano regolarmente la sera e nei fine settimana per far fronte alle grandi richieste date dal loro lavoro (Gill, 2010: 235) e non si avvalgono per intero del loro diritto alle ferie annuali (Crang, 2007, 510).

\section{Management}

Negli ultimi decenni è stato attuato un «nuovo managerialismo» negli istituti di istruzione superiore. Questo nuovo managerialismo può essere inteso come l'adozione di forme organizzative, tecnologie, pratiche di controllo manageriale e ideologie dell'impresa privata applicate a organizzazioni del settore pubblico come le università (Deem et al., 2007, 24-28). In condizioni neoliberali e post-fordiste, le università britanniche sono gestite sempre più spesso secondo modalità aziendali. Le funzioni accademiche vengono pertanto suddivise in processi controllabili (Lorenz, 2012, 610) che portano a una frammentazione dei profili professionali. Lo stile di gestione del settore privato porta alla creazione di una struttura organizzativa gerarchica, alla divisione e standardizzazione del lavoro, alla specializzazione ristretta e alla routinizzazione dei compiti per aumentare la responsabilizzazione e la misurazione da parte del management. Prichard e Willmott (1997) hanno sottolineato che le università stanno implementando molti elementi di «managerialismo soft», sollecitando gli accademici a raggiungere gli obiettivi di performance e incoraggiando così l'autodisciplina senza dover attuare "hard management". A causa della pressione a raggiungere gli obiettivi di performance, stanno diminuendo le risorse individuali per partecipare attivamente al processo decisionale a livello istituzionale e scolastico: 
Eppure, in realtà, l'aumento del managerialismo implica che il contributo del personale al processo decisionale passi da una forma di partecipazione collegiale ad un ruolo che è, nel migliore dei casi, consultivo, in cui il personale accetta e sostiene volontariamente i capi dipartimento che poi gestiscono il processo attraverso il quale le risorse vengono acquisite e assegnate. (Willmott, 1995, 996)

Tancred-Sheriff $(1985,384)$ ha paragonato il processo decisionale nelle università ad un «volante per bambini nella macchina di papà» dove un mucchio di comitati e gruppi, pur dotati formalmente di poteri decisionali, sono in realtà relativamente privi di potere. Più di 20 anni fa, Prichard e Willmott (1997) hanno condotto 36 interviste con alti funzionari quali rettori, decani, direttori di istituto e direttori di dipartimento di quattro università britanniche a proposito della loro concezione del managerialismo. Gli autori hanno riferito che i loro intervistati "hanno parlato dell'attuazione di iniziative strategiche, della gestione del personale, dell'assunzione di responsabilità e persino dell'essere un piccolo imprenditore» (Prichard e Willmott, 1997, 313). Miller (1991, 111) sosteneva che i rettori tendevano ad agire come amministratori delegati.

\section{Meccanismi di controllo}

Sebbene le procedure di sorveglianza, di monitoraggio e di audit non siano nuove come meccanismi di controllo all'interno delle università (ad esempio, il Research Assessment Exercise ${ }^{2}$ è in vigore fin dagli anni '80), e sebbene l'università non sia l'unico, e nemmeno il più estremo tra i

2 Il Research Assessment Exercise era un sistema di valutazione della qualità della ricerca svolta nelle università del Regno Unito che si basava su un insieme di indici, tra cui numero e prestigio delle pubblicazioni. Sulla base di queste valutazioni, vengono stilate delle classifiche delle università e vengono distribuiti fondi pubblici. A partire dal 2014 è stato sostituito dal Research Excellence Framework (cfr. nota 5) (nota della traduttrice). luoghi dove si pratica la sorveglianza, tali procedure stanno prendendo piede in modo significativo negli istituti di istruzione superiore del Regno Unito da alcuni anni (Burrows, 2012, 357). I metodi di misurazione funzionano a più livelli, come ad esempio a livello istituzionale, nazionale e internazionale, ma il singolo accademico deve confrontarsi con ciascuna di queste (Burrows, 2012, 359). Nelle università esiste una complessa serie di procedure di monitoraggio e di misurazione, tra cui finanziamenti ricevuti, indice di citazioni, modelli di carico di lavoro, dati trasparenti sui costi, «eccellenza» della ricerca, valutazione degli studenti, capacità di creare occupazione, fattori di impatto e classifiche commerciali delle università (De Angelis e Harvie, 2009, 11-14). Burrows (2012, 359) ha scoperto che gli accademici britannici attualmente sono soggetti a più di $100 \mathrm{mi}$ sure e indici diversi. Gli accademici sono misurati individualmente rispetto ad altri colleghi, così come raggruppati e misurati rispetto ad altri gruppi per valutare e classificare i rispettivi valori accademici. Gill (2014, 22-24) sostiene che la cultura della sorveglianza e i regimi di audit portano alla creazione nelle università di una nuova psicologia e di nuove strutture del sentimento caratterizzate da pressioni individuali, ansia e minacce. Il proliferare delle classifiche ha innescato una cultura della stigmatizzazione che si traduce in auto-sorveglianza:

\begin{abstract}
L'essere soggetti che lavorano, si auto-motivano e prendono l'iniziativa è ciò che fa del mondo accademico l'emblema perfetto di questo periodo neoliberale, ma fa anche parte di un paesaggio mentale in cui il non avere successo [...] è erroneamente riconosciuto [...] come un fallimento (morale) dell'individuo. (Gill, 2010, 240)
\end{abstract}

\section{Salute mentale e fisica}

Diverse ricerche empiriche hanno studiato i problemi di salute mentale e fisica presenti nelle istituzioni di istruzione superiore. In una indagine della University and College Union $(2014,2)$ il $60 \%$ degli 
intervistati ha dato segni di un certo livello di disagio psicologico. Secondo Watts e Robertson (2011) il livello di burnout tra i membri del corpo docenti delle università è paragonabile a quello di categorie «a rischio» come i professionisti della sanità. Il disagio psicologico degli accademici è maggiore di quello riportato da altre categorie professionali ed è causato da fattori quali il forte conflitto tra vita professionale e vita privata (Kinman e Wray, 2013, 6). Il lavoro accademico, in senso stretto e in senso lato, tende a «invadere il contesto domestico sia fisicamente (per esempio con il lavoro da casa alla sera o nei weekend), sia psicologicamente (preoccupazione per i problemi di lavoro, disturbi del sonno, stato di irritabilità con familiari e amici)» (Kinman e Wray, 2013, 7).

\section{Genere}

Insieme ad altre caratteristiche come la classe sociale, l'etnia, l'età e la disabilità, il genere contribuisce a plasmare le esperienze dei lavoratori del mondo accademico. Complessivamente, ci sono 273895 accademici (personale accademico a tempo pieno e a tempo parziale e personale accademico impiegato in modo atipico) nel settore dell'istruzione superiore del Regno Unito. Complessivamente, il 37\% del personale accademico a tempo pieno ha uno stipendio compreso tra 43325 e 58172 sterline. Tra il personale accademico a tempo pieno, una proporzione maggiore di uomini (25.3\%) ha uno stipendio pari o maggiore di 58.172 sterline, rispetto alle donne (13.9\%). La percentuale di donne accademiche è del 45,0\%. Per il personale accademico a tempo pieno, la percentuale di donne è del $40,0 \%$ e per il tempo parziale del 55,1\%. Circa il $47,2 \%$ del personale accademico impiegato in modo atipico è costituito da donne, nonostante il fatto che le donne impiegate in ambito accademico siano meno numerose rispetto agli uomini. Inoltre, quanto più alta è la posizione nella gerarchia dell'istruzione superiore, meno donne si trovano. Per esempio, il 56,2\% degli studenti nel Regno Unito sono donne, ma solo il 23,1\% dei professori sono donne (tutti i dati per l'anno accademico 2014/2015: Higher Education Statistics Agency, 2016).

\section{Metodologia}

Questo articolo si basa su un piccolo studio di caso per il quale ho condotto dieci interviste qualitative, semi-strutturate, di persona, con accademici. L'attenzione si è concentrata su persone impiegate presso gli istituti di istruzione superiore «atipicamente», per esempio con un contratto a tempo determinato, un contratto occasionale, con retribuzione a ore o con un contratto a zero ore ${ }^{3}$. I partecipanti sono stati scelti tra nove diverse università (cinque università pre-1992, e quattro post-1992) in tutta la Scozia, utilizzando una strategia di campionamento a quote (Lune e Berg, 2017, 39). Le variabili utilizzate per stabilire le quote comprendevano il genere, il livello di istruzione, gli istituti di istruzione superiore, le condizioni di lavoro e l'età. Gli intervistati sono stati individuati mediante i siti web delle università, non avevano alcun rapporto personale con l'intervistatore e sono stati contattati direttamente via e-mail. Le interviste hanno avuto luogo in uffici, aule universitarie e locali pubblici nel corso del 2016. Dopo aver ottenuto il consenso, le interviste sono state audio-registrate e completamente trascritte. Ai partecipanti è stata data la libertà di abbandonare l'intervista in qualsiasi momento. Tutti i dati sono stati conservati in modo sicuro, trattati in modo confidenziale e anonimo. Le interviste hanno avuto una durata compresa tra i 50 e i 100 minuti. Una copia della trascrizione è stata inviata ai partecipanti per ulteriori commenti e per approvazione finale. I testi sono stati analizzati con il supporto dell'analisi di contenuto (Krippendorff, 2004; Lune e Berg, 2017) e

3 Il contratto a zero ore è una tipologia di contratto in uso nel mondo accademico britannico, e non solo, in base al quale il datore di lavoro può ingaggiare il lavoratore/la lavoratrice senza dover garantire un numero minimo di ore di impiego e con un preavviso minimo (nota della traduttrice). 
per trovare risposte alle mie domande su come gli accademici sentano che le loro condizioni di lavoro vengano modellate dal contesto politico ed economico.

I profili socio-demografici degli intervistati sono riportati nella tabella 1 . Diversi autori hanno già condotto lavori empirici in questo contesto (es. Prichard eWillmott, 1997; Deem et al., 2007; Archer, 2008; Norkus, Besio e Baur, 2016). Il mio studio mira a contribuire a questo corpus di conoscenze concentrandosi su accademici che sono impiegati precariamente in istituti di istruzione superiore attraverso un'analisi approfondita dei significati, delle identità e delle esperienze degli individui, il ché favorisce un approccio qualitativo, piuttosto che quantitativo (Gray, 2004, 22).

Tabella 1: Profili socio-demografici degli intervistati $(n=10)$

\begin{tabular}{|c|c|}
\hline Genere & Sei donne, quattro uomini \\
\hline Nazionalità & $\begin{array}{l}\text { Sette britannici, un/a tedesco /a, un/a } \\
\text { austriaco /a, un/a belga }\end{array}$ \\
\hline Livello di istruzione & Dieci dottori di ricerca \\
\hline Area disciplinare & $\begin{array}{l}\text { Educazione (uno/a), scienze politiche } \\
\text { (uno/a), scienze sociali (due), psicologia } \\
\text { (uno/a), sociologia (due), informatica } \\
\text { (uno/a), economia (uno/a), scienze della } \\
\text { salute (uno/a) }\end{array}$ \\
\hline $\begin{array}{l}\text { Istituzione di } \\
\text { istruzione superiore }\end{array}$ & $\begin{array}{l}\text { Sei università pre-1992 (due del Gruppo } \\
\text { Russell)a, quattro università post-1992 }\end{array}$ \\
\hline Tipo di contratto & $\begin{array}{l}\text { Sette a tempo pieno, uno / a a tempo } \\
\text { parziale, uno / a con retribuzione a ore }\end{array}$ \\
\hline Durata del contratto & $\begin{array}{l}\text { Nove a tempo determinato (uno / a in } \\
\text { congedo), un / a con contratto a tempo } \\
\text { determinato per l' } 80 \% \text { e a tempo in- } \\
\text { determinato per il } 20 \%\end{array}$ \\
\hline Età & $\begin{array}{l}\text { Età minima } 33 \text { anni, età massima 56, } \\
\text { età media } 42 \text { anni }\end{array}$ \\
\hline
\end{tabular}

a II Gruppo Russell è costituito da ventiquattro università britanniche che, in virtù delle loro performance nelle classifiche di valutazione della qualità della ricerca, ricevono oltre due terzi del totale dei finanziamenti pubblici nel Regno Unito (nota della traduttrice).

\section{Analisi e interpretazione}

Questa sezione presenta e discute alcuni risultati riguardanti l'insicurezza occupazionale, il carico di lavoro, il management, i meccanismi di controllo, la salute mentale e fisica e il genere. Queste sono alcune delle dimensioni che sono state discusse nelle interviste che ho condotto. Per una panoramica strutturale del questionario dell'intervista, si veda Allmer (2018).

\subsection{Insicurezza occupazionale}

Quando è stato chiesto loro che peso avesse l'insicurezza professionale nell'attuale posto di lavoro, i partecipanti hanno espresso apprensione e preoccupazioni circa la loro situazione di insicurezza e hanno riferito di provare sentimenti di precarietà, mancanza di prospettive, crescente competitività e necessità di presentare molteplici candidature per nuovi posti di lavoro. La maggior parte dei partecipanti era preoccupata per la natura precaria del loro lavoro e aspirava alla sicurezza economica. «Volevo davvero una posizione più sicura», ha affermato Partecipante 2. Questo punto di vista è stato ripreso da Partecipante 6 che ha detto: «Preferirei avere una posizione permanente e smettere di chiedermi dove sarò tra cinque anni». Una ricercatrice post-doc ha sottolineato come la sua situazione lavorativa insicura fosse causa di depressione, la facesse sentire svalutata e ne influenzasse negativamente l'autostima. Un'altra ricercatrice mi ha detto che non riusciva più a concentrarsi sul suo lavoro a causa della situazione lavorativa insicura: la precarietà del lavoro la preoccupava ed era costantemente nei suoi pensieri.

Gli intervistati hanno anche descritto salari inadeguati e insicurezza economica. Un assegnista con incarichi di insegnamento, che coordinava un corso di studio del suo dipartimento, mi ha detto che gli assegnisti sono pagati con un salario di livello 7, anche se molti di loro hanno responsabilità e impegni destinati al personale di livello 8, come, per esempio, organizzare percorsi formativi, insegnare o dirigere corsi di studio. Ha proseguito dicendo che gli assegnisti con incarichi di insegnamento svolgono mansioni di livello 8 , senza essere adeguatamente inquadrati contrattualmente né retribuiti (questi gradi si riferiscono a ruoli accademici e profili validi nelle università britanniche). Una giovane accademica ha riferito che i suoi precedenti dipartimenti volevano che lei svolgesse degli incarichi non retribuiti: 
Gli altri dipartimenti per cui lavoravo volevano che continuassi a fare lezioni e valutazione di esami, ma non mi avrebbero pagato. E' arrivato un momento in cui ho detto «no». No, non lo faccio più». Mi hanno intervistato per un lavoro come docente, non mi hanno dato il lavoro, ma volevano comunque che tenessi lezioni di tanto in tanto e con il relativo lavoro di correzione [...] ma non mi hanno nemmeno offerto una retribuzione a ore. (Partecipante 2)

Una docente con retribuzione a ore aveva altri due lavori oltre a quello principale per poter avere una certa sicurezza economica e per poter restare nell'ambito dell'istruzione superiore. Ha descritto la sua intenzione di scrivere articoli durante le vacanze estive non retribuite per poter essere più competitiva nel mercato del lavoro accademico. Gli intervistati hanno anche sostenuto che il tempo dedicato alla preparazione delle lezioni non era adeguatamente riconosciuto a livello retributivo. Gli intervistati hanno detto di doversi confrontare, nel loro lavoro, con una mancanza di informazioni, indicazioni confuse e mancanza di prospettive. Un docente era confuso sulle sue prospettive all'interno del proprio istituto:

Per tutto il mese di gennaio e febbraio, ci è stato detto «È fantastico, renderanno tutti a tempo indeterminato, c'è una reale volontà di sbarazzarsi di questi contratti temporanei» e poi ci hanno detto l'esatto contrario. (Partecipante 1)

Una ricercatrice ha detto di sentirsi estremamente insicura e di non sapere cosa sarebbe successo al termine della sua attuale borsa di studio. Un docente con retribuzione oraria era consapevole del fatto che non avrebbe ottenuto alcuna informazione sulla proroga del contratto fino a poco prima dell'inizio del semestre successivo. Una ricercatrice a tempo determinato mi ha detto di sentirsi insicura e che l'insicurezza derivava dal non sapere quando e da dove sarebbe potuto venire il successivo contratto, quale percentuale di una posizione a tempo pieno si sarebbe potuta as- sicurare e quando un particolare progetto sarebbe potuto cominciare. Gli intervistati si sono sentiti spinti ad essere competitivi e a lavorare intensamente in ogni momento a causa della natura temporanea del loro lavoro e della relativa insicurezza.

Allo stesso tempo, questi intervistati erano consapevoli e preoccupati dell'atmosfera competitiva tra il personale. Gli intervistati mi hanno detto che la concorrenza e la pressione per i posti di lavoro permanenti e sicuri crescono costantemente, cosa che loro trovavano difficile a livello individuale.

I partecipanti hanno menzionato $\mathrm{i}$ loro timori e le loro preoccupazioni, come accademici, di rimanere disoccupati e del rischio di essere licenziati. Un docente ha affermato che, qualora il numero di studenti nel suo college fosse diminuito, gli accademici a tempo determinato, compreso lui, sarebbero stati i primi ad essere licenziati. Una giovane ricercatrice ha descritto come trovasse davvero preoccupante l'idea di essere disoccupata una volta terminato il suo dottorato. Molti intervistati hanno dichiarato di monitorare costantemente le offerte di lavoro e di fare domanda per nuovi posti. Un docente di un'università del Gruppo Russell ha detto che, come accademici a tempo determinato, si è costantemente alla ricerca di qualcos'altro. "Più a lungo rimaniamo assegnisti con incarichi di insegnamento, meno risultati di ricerca produciamo e più diventa difficile competere con le persone che sono all'esterno, che hanno già posti professorali» (Partecipante 1). Altri si sono lamentati del fatto che trovare un nuovo lavoro richiede tempo ed energia, è faticoso e umiliante:

È solo uno spreco di tempo. È faticoso. È [...] Non so se posso dire, è anche umiliante [...] E quando non si tratta di preparare candidature di lavoro, si tratta di scrivere domande di finanziamento per progetti e io vorrei semplicemente fare qualcos'altro. (Partecipante 6)

I partecipanti hanno anche descritto quanto fosse difficile per loro trovare il 
tempo per scrivere le candidature di lavoro e prepararsi adeguatamente ai colloqui.

\subsection{Carico di lavoro}

Molti partecipanti hanno riferito di un carico di lavoro eccessivo nei loro ambiti. Un docente ha detto di aver dovuto scrivere valutazioni per più di 20 tesi di laurea, in aggiunta ad un pesante carico di lavoro di insegnamento. Ha continuato dicendo che anche il lavoro di tutoring individuale degli studenti era un onere molto rilevante. Un'altra intervistata mi ha detto che lavorare con contratti a retribuzione oraria per diversi dipartimenti contemporaneamente era il lavoro più duro che avesse mai fatto:

Perché stavo cercando di lavorare in più dipartimenti diversi, perché non avevo un salario fisso, ero pagata a ore, dovevo coordinare il corso ed ero pagata l'equivalente di una sola ora per tutta la fase di preparazione [...] Penso di non aver mai lavorato tanto quanto allora. (Partecipante 2)

Una ricercatrice a progetto si è dovuta confrontare con un elevato carico di lavoro nel suo progetto e si è sentita particolarmente sotto pressione perché ha dovuto affrontare il doppio peso di avere un bambino e lavorare a tempo pieno. Un'accademica più anziana di un'università post-1992 mi ha detto che le persone erano sovraccaricate di lavoro e di oneri e tendevano ad essere maniaci del lavoro, ma che lei, più invecchiava, meno era disposta a lavorare fuori orario. Un giovane ricercatore sapeva di lavorare «più del normale» (Partecipante 6), ma lo considerava necessario. Un'altra intervistata ha detto che c'erano alti e bassi in termini di carico di lavoro nei suoi progetti, ma ha sottolineato quanto questo aspetto fosse difficile da controllare individualmente come ricercatori a contratto. Ha proseguito dicendo che «si fa costantemente affidamento su altre persone». Molti dei partecipanti si sono lamentati della pressione del lavoro e di un alto livello di stress. Come ha raccontato un assegnista con incarichi di insegnamento, una grande fonte di stress è data dai tempi stret- ti concessi per la correzione delle prove scritte. Ha aggiunto che, nel dipartimento in cui era impiegato, era considerato normale fare gli straordinari.

Un'altra intervistata ricordava un periodo durante il quale vedeva alcuni degli studenti più spesso dei suoi stessi familiari e difficilmente riusciva a trovare il tempo per mangiare e dormire bene. Un ricercatore a contratto ha descritto quanto i progetti di ricerca possano essere fonte di stress e di pressione, soprattutto nelle loro fasi finali. Un'altra ricercatrice a progetto ha sostenuto che i responsabili dei progetti tendono a promettere molto nella domanda di finanziamento e che questo può portare a un ambiente di lavoro molto stressante per l'intero team di ricerca. Ha poi spiegato come il fatto di essere impegnati in modi diversi su diversi progetti contemporaneamente può portare a un livello di stress molto elevato. Un assegnista con incarichi di insegnamento ha detto di aver cercato di evitare di lavorare nei fine settimana, ma che questo lo ha portato a lavorare a lungo la sera durante la settimana; doveva comunque lavorare da casa di tanto in tanto per restare al passo con tutto. Come ha detto un altro intervistato, "Non c'è tempo per i fine settimana» (Partecipante 2). Una giovane madre ha raccontato di lavorare spesso la sera, dopo essere tornata a casa da una giornata lavorativa di 8 ore.

Molti dei partecipanti avevano spazi di lavoro condivisi e tendevano a lavorare da luoghi diversi, tra cui l'ufficio, i bar e l'abitazione privata. Mentre alcuni apprezzavano questa flessibilità, altri erano preoccupati per la confusione che essa generava tra la vita lavorativa e la vita privata. I partecipanti hanno riferito di avere difficoltà nello sfruttare completamente il loro diritto alle ferie annuali a causa del pesante carico di lavoro. Un intervistato non è mai riuscito a prendere tutte le ferie annuali e ha trovato difficile ottenere ferie allo stesso momento del partner. Un'altra intervistata ha confessato di non aver mai preso ferie annuali perché sentiva che «qualcosa ne avrebbe risentito" (Partecipante 2). Un ricercatore a contratto ha detto che è difficile prendere le ferie annuali se si lavora 
su tre diversi progetti. C'è sempre almeno uno di questi che ha bisogno di te in quella particolare settimana:

Non è facile quando si ha più di un contratto prendere le ferie annuali, ad esempio [...] Se si hanno contratti multipli, ce ne sarà sempre uno che ha davvero bisogno che tu faccia qualcosa questa settimana, o quella successiva. (Partecipante 9)

\subsection{Management}

Agli intervistati è stato chiesto cosa pensassero del management del loro dipartimento. Molti di loro hanno riferito esperienze negative con uno stile di management autoritario, con la mancanza di supporto da parte del personale più anziano e di aver rinunciato ad essere ambiziosi e leali nei confronti del dipartimento. Una ricercatrice post-doc ha parlato di esperienze negative con uno stile di management autoritario nel suo posto di lavoro precedente. Ha descritto come i responsabili del management portassero molta negatività e come questo abbia spinto alcune persone ad andarsene. Una giovane ricercatrice ha detto di non sentirsi sostenuta dal personale più anziano in termini di avanzamento di carriera. Questa mancanza di supporto è stata riferita anche da altri intervista$\mathrm{ti}$, specialmente in riferimento al lavoro di ricerca e allo sviluppo della carriera. Il managerialismo, le strutture organizzative gerarchiche, la specializzazione ristretta e le mansioni di routine hanno portato frustrazione e rabbia a questi partecipanti. Un professore mi ha detto che non vedeva più la necessità di essere leale e ambizioso, poiché il suo contratto non era stato reso permanente:

Non avevano intenzione di rinnovarmi il contratto, e io non sono più sicuro di quanto mi prema dimostrare che sono super leale, grande lavoratore, e persino ambizioso. (Partecipante 1)

Analogamente, una madre single ha raccontato di essersi impegnata molto in un progetto senza ottenere alcun riconosci- mento, e che questo l'ha portata a rinunciare completamente ad essere leale.

\subsection{Meccanismi di valutazione}

Ai partecipanti sono state chieste informazioni sulle loro esperienze in relazione ai meccanismi di controllo delle loro prestazioni accademiche. Hanno parlato dei processi di revisione annuale, del Research Excellence Framework (REF) ${ }^{4}$ e di «stare al gioco». Un giovane accademico mi ha detto che molte persone nel suo dipartimento considerano la revisione annuale delle prestazioni e dell'avanzamento come un'attività puramente manageriale, un esercizio irrilevante che non rispecchia il loro lavoro o i loro interessi. La maggior parte dei partecipanti non era stata soggetta alla valutazione del REF, ma conosceva bene la procedura. Un ricercatore post-doc pensava che il REF fosse un meccanismo ingiusto e inaffidabile, specialmente nei confronti di coloro che hanno incarichi di insegnamento. Un ricercatore sosteneva che il REF non fosse obiettivo e che privilegiasse persone di fama:

\begin{abstract}
Se hai già un nome, una reputazione, i tuoi articoli avranno più probabilità di essere pubblicati nelle riviste con 3 o 4 stelle e dunque tu avrai più possibilità di ricevere finanziamenti. (Partecipante 7)
\end{abstract}

Riteneva inoltre che il REF rendesse svantaggioso condurre studi storico-longitudinali e che contribuisse a creare una cultura della burocratizzazione. "Tutti stanno al gioco qui», mi ha detto un giovane ricercatore di un'università del Gruppo Russell, che criticava queste tendenze ma ammetteva anche di farne parte. Quando un docente più anziano ha riferito delle sue conversazioni con i giovani accademici, ha detto che le persone sono coscienti delle

4 Il Research Excellence Framework (REF) è un processo di valutazione che valuta la ricerca degli istituti di istruzione superiore nel Regno Unito. Con alcune eccezioni, si svolge ogni sei anni e viene utilizzato per decidere l'ammontare del finanziamento pubblico della ricerca per le singole università (nota dell'autore). 
contraddizioni insite nel sistema di istruzione superiore, ma che non hanno molte speranze di cambiamento politico al momento, il ché porta ad una cultura dello «stare al gioco»:

Le persone sanno che stanno succedendo tutte queste cose, ma allo stesso tempo non hanno molta speranza che le cose cambino, così provano in qualche modo a stare al gioco. (Partecipante 1)

\subsection{Salute fisica e mentale}

Alla domanda su quanto le condizioni di lavoro incidano sulla loro salute mentale e fisica, gli intervistati hanno riferito disagio psicologico ed emotivo, limitata vita sociale, tensioni nei rapporti di lavoro e mancanza di riconoscimento. Una ricercatrice mi ha detto di essere sempre stanca e nervosa e di aver avuto momenti in cui non riusciva né a dormire né a mangiare correttamente a causa dello stress e del lavoro eccessivo. Ha continuato dicendo che l'insegnamento era costantemente nei suoi pensieri prima di andare a dormire e non riusciva a sbarazzarsene. Anche un'altra intervistata ha detto che il lavoro è una sorta di retro-pensiero che rimane sempre nella testa. Un'altra ha riferito di aver vissuto in passato periodi di stress e di esaurimento che avevano influenzato anche le sue condizioni di salute. Un giovane ricercatore associato di un'università del Gruppo Russell si è lamentato del fatto che "avere una posizione precaria ha avuto un impatto negativo anche sulla mia relazione, perché sono amareggiato e non sono la persona più piacevole con cui convivere in questo momento» (Partecipante 6). Un altro intervistato ha affermato di sentirsi meglio in ufficio, mentre di essere preoccupato e stressato quando non al lavoro:

Quando sono via [...] sono più stressato [...] penso al lavoro di più, tipo, e sono preoccupato di essere via, mi preoccupa [...] quando vado in ufficio [...] mi sento meglio di quando non ci vado [...] anche se ho preso un congedo annuale. (Partecipante 10)
Un altro intervistato, parlando della fase finale del suo dottorato di ricerca, che aveva dovuto scrivere mentre allo stesso tempo iniziava a insegnare all'università, ha detto: "Non avevo una vita sociale, non c'era una vita sociale» (Partecipante 10). Un docente retribuito a ore ha parlato di come la sua vita sociale si fosse ridotta e di come lo stress e l'eccesso di lavoro gli avessero causato sofferenze. Un giovane accademico belga mi ha detto che, a causa del suo lavoro precario, temporaneo e sempre in mobilità, non si è nemmeno registrato presso il Servizio Sanitario Nazionale (NHS). Un'altra partecipante ha affermato di essere riuscita a raggiungere un discreto equilibrio tra vita lavorativa e vita personale, ma che questo probabilmente era il motivo per cui non era riuscita ad ottenere un contratto a tempo indeterminato:

\begin{abstract}
Di solito non lavoro nei fine settimana, non sono una maniaca del lavoro. Tendo ad avere un buon equilibrio tra famiglia e lavoro, ma questo è probabilmente anche il motivo per cui ho ancora un contratto a tempo determinato e non ho una posizione permanente (ride). (Partecipante 5)
\end{abstract}

Un anziano docente di un'università del Gruppo Russell ha descritto una situazione nel suo dipartimento dove c'erano tensioni tra i dipendenti, dove le persone erano divise in diversi fronti e le questioni fondamentali rimanevano irrisolte. Un ricercatore a contratto ha evidenziato come i professori, e specialmente quelli di nuova nomina, tendevano a trattare i ricercatori più giovani che collaboravano ai loro progetti in modo paternalistico, il ché poteva portare a tensioni e a complicare le cose. La precarietà del lavoro, l'obbedienza alle strutture organizzative gerarchiche e il costante adempimento di compiti ristretti hanno avuto un impatto anche sulla percezione dell'identità e sul riconoscimento degli accademici. Una ricercatrice ha detto che ci si può sentire sminuiti se viene richiesto di lavorare secondo un orario prestabilito e se viene concessa poca autonomia nel lavoro. Ha continuato dicendo che era difficile per un'assegnista di ricerca es- 
sere presa sul serio. Un'altra intervistata ha sostenuto che i tutor non solo sono pagati poco, ma non ottengono nemmeno il riconoscimento che meritano.

\subsection{Genere}

Interrogate sulle questioni di genere, le ricercatrici mi hanno informato che il mondo accademico porta sia flessibilità che insicurezza, in particolare per le donne. Le partecipanti hanno riconosciuto le discriminazioni di genere nel mercato del lavoro e hanno sostenuto che è ancora più difficile per le donne avere successo nell'istruzione superiore. Questa percezione è rafforzata dalle statistiche, che mostrano che più in alto si guarda nella gerarchia dell'istruzione superiore, tante meno donne si trovano; ad esempio, le donne rappresentano solo il 23,1\% dei professori nel Regno Unito nell'anno accademico 2014/2015 (Higher Education Statistics Agency, 2016). Poiché le donne hanno maggiori probabilità di essere impiegate in modo precario nell'ambito dell'istruzione superiore, ho deciso di condurre la maggior parte delle mie interviste con donne accademiche, per restituire meglio il quadro generale. La maggior parte delle intervistate doveva anche occuparsi dei figli, cosa che è probabilmente legata alla giovane età delle partecipanti, che è a sua volta legata alla loro posizione lavorativa precaria. In questa sezione, presenterò alcune citazioni da queste interviste, senza voler suggerire che la disuguaglianza di genere nell'ambito dell'istruzione superiore riguarda solo le madri di bambini piccoli.

Una ricercatrice con un figlio di 6 mesi mi ha detto che le persone sono costantemente preoccupate che tu possa prendere un congedo di maternità. Ha anche sentito alcuni colleghi parlare della preoccupazione che una candidata a un posto di lavoro potesse essere incinta:

Negli ultimi colloqui svolti qui in dipartimento, dovevano assumere tre nuovi docenti, e io so che avevano dei timori nei confronti di una di loro. Lei è venuta per il colloquio e temevano potesse essere incinta [...] Ho sentito persone parlare di questo timore. (Partecipante 4)
Un'altra accademica ha fatto un'osservazione simile, dicendo che come ricercatrice a contratto è molto difficile ottenere un lavoro ad una certa età, perché i direttori dei progetti di ricerca temono che si possa rimanere incinta durante il progetto. Una giovane ricercatrice ha affermato di non poter essere competitiva, perché doveva occuparsi di suo figlio da sola e si sentiva quindi svantaggiata in ambito accademico:

\begin{abstract}
Sono una madre single [...] Non sento di poter essere competitiva quanto altri [...] Mi sento svantaggiata.[...] Ci si sente molto limitate nelle proprie possibilità [...] Così come è questa insicurezza, è proprio come un circolo vizioso perché devi mantenere questi contratti a tempo e precari, perché non puoi competere ad un livello che ti permetta di ottenere qualcosa di permanente. È qualcosa che [...] si perpetua. (Partecipante 8)
\end{abstract}

Una docente donna ha sostenuto che gli uomini tendono ad essere più bravi sia a vendersi che a presentarsi nell'ambiente accademico. Una giovane ricercatrice ha detto di non sentirsi a suo agio ad esprimere la propria opinione in un gruppo di ricerca dove prevalevano gli uomini e di sentirsi perciò messa a tacere.

\section{Riepilogo}

Questo articolo si è occupato di alcune delle basi teoriche del lavoro accademico e delle condizioni di lavoro nelle università. Ha anche presentato e discusso i risultati di una ricerca qualitativa in relazione alla precarietà del lavoro, al carico, alla gestione, ai meccanismi di controllo, alla salute mentale e fisica e al genere. I risultati principali possono essere riassunti come segue.

Insicurezza occupazionale. La ricerca conferma i risultati di altri studiosi secondo i quali c'è una tendenza alla precarizzazione e alla temporaneità dell'impiego nell'istruzione superiore. I contratti a tempo determinato tendono ad avere un impatto sui dipendenti a 
molti livelli. I partecipanti a questo studio menzionano preoccupazioni e paure per la loro situazione di insicurezza e riferiscono di precarietà, mancanza di prospettive, retribuzione inadeguata, aumento della competizione e continua ricerca di nuovi posti di lavoro.

- Carico di lavoro. Anche questi risultati confermano le ricerche precedenti, secondo le quali gli accademici tendono ad avere confini fluidi tra lo spazio di lavoro e gli altri spazi della vita umana, tra il tempo di lavoro e il tempo libero e che le culture della connessione costante hanno trasformato l'università in una "fast-accademia". Nel mio studio, la maggior parte degli intervistati ha riferito di carichi di lavoro e di impegni eccessivi nel loro ambiente di lavoro. Alcuni intervistati erano anche preoccupati per il confine sempre più sfumato tra lavoro e vita privata.

, Management. Secondo la letteratura, le università sono sempre più gestite secondo un modello aziendale, descritto come "nuovo managerialismo», che risponde alle condizioni post-fordiste. Le attività accademiche sono così suddivise in processi controllabili. Questo studio fornisce un'ulteriore prova di ciò: $\mathrm{i}$ partecipanti hanno riferito di esperienze negative con uno stile di management autoritario, la mancanza di sostegno da parte del personale più anziano e la rinuncia ad essere leali e ambiziosi. Il managerialismo, le strutture organizzative gerarchiche, la specializzazione ristretta e i compiti di routine hanno portato molti degli accademici intervistati a provare frustrazione e rabbia.

, Meccanismi di valutazione. Questa ricerca chiarisce ulteriormente il modo in cui i sistemi di misurazioni operanti a livello istituzionale, nazionale e internazionale hanno un impatto sui singoli lavoratori accademici, con gli intervistati che descrivono le loro esperienze negative dei processi di revisione annuale e del REF e parlano di «stare al gioco».

, Salute fisica e mentale. Il disagio psicologico degli accademici supera molti altri gruppi professionali ed è causato da fattori quali un alto livello di conflitto tra lavoro e vita privata. Ciò emerge anche in questo studio dalle descrizioni fatte dagli intervistati del loro disagio psicologico ed emotivo, della vita sociale limitata, dei rapporti di lavoro tesi e del mancato riconoscimento.

, Genere. Infine, questo studio conferma risultati precedenti relativi alla discriminazione di genere nell'istruzione superiore, con le intervistate che hanno descritto le sfide aggiuntive che devono affrontare nel mercato del lavoro e i compromessi che devono compiere per combinare il lavoro con la genitorialità. Mentre alcune hanno scelto il settore accademico perché sembra offrire una maggiore flessibilità rispetto ad altre forme di lavoro, per questo hanno pagato un prezzo elevato in termini di insicurezza.

\section{Conclusione}

Le università giocano un ruolo centrale nel capitalismo informazionale. Tuttavia, gli istituti di istruzione superiore hanno subito trasformazioni economiche, politiche $\mathrm{e}$ culturali che hanno portato a competizione, orientamento al mercato e nuove forme di management. Questi cambiamenti hanno effetti su molti livelli, comprese le condizioni di lavoro e le pratiche delle persone coinvolte nel processo di acquisizione delle informazioni. Questo articolo mirava a comprendere come le condizioni di lavoro e le pratiche esistenti nelle università formino i significati, le identità e le esperienze degli individui, concentrandosi sugli accademici impiegati in modo precario. Le basi teoriche e i risultati empirici suggeriscono che gli accademici precari si sentono insicuri e sovraccarichi di lavoro e che sperimentano nuove forme di managerialismo e meccanismi di controllo che hanno un impatto sulla loro salute fisica e mentale. Tuttavia, quando si tratta di politica, di lotta e di trovare alternative, è necessario che la ricerca futura affronti anche altre questioni, in modo sia teorico che empirico. Quali sono le situazioni politiche reali e potenziali nelle università 
per quanto riguarda i temi della solidarietà, della partecipazione e della democrazia? Quali sono le sfide per rivendicare l'università come luogo di lotta? Fino a che punto la lotta nelle università può essere collegata a rivendicazioni sociali più ampie? Come si presentano le potenziali alternative politiche dentro e fuori il contesto dell'istruzione superiore? Sebbene queste domande non rientrassero nell'ambito affrontato da questo articolo, esse aiuterebbero a trovare strategie per superare le sfide che gli accademici si trovano oggi ad affrontare, come è stato analizzato qui.

\section{Profilo biografico della traduttrice Gloria Dagnino}

Gloria Dagnino is Responsible for the Equal Opportunities Service of the Università della Svizzera italiana (USI) in Lugano, Switzerland. She is also coordinator and main lecturer of the Film economics module that the Institute of Media and Journalism at USI offers within the Master Réseau Cinema $\mathrm{CH}$. Previously, she has been visiting SNF research fellow at the University of East London and invited lecturer at the Université de Strasbourg. Her research interests include political economy of media, media and advertising convergence, Italian cinema, audiovisual media labour and diversity in the film industry. Her first book, on branded entertainment and Italian cinema, is forthcoming from Routledge. In 2016, she obtained her PhD in Communication from USI.

\section{Bibliografia}

Allmer, T. (2017). Academic labour, digital media and capitalism. Critical Sociology. Advance online publication. doi:10.1177/0896920517735669.

Allmer T. (2018). Precarious, always-on and flexible: A case study of academics as information workers. European Journal of Communication. Advance online publication. doi:10.1177/0267323118783794
Archer, L. (2008). The new neoliberal subjects? Young/er academics' constructions of professional identity. Journal of Education Policy, 23, 265-285.

Bryson, C. e N. Barnes (2000). The casualisation of employment in higher education in the United Kingdom. Academic Work and Life, 1, 187-241.

Burrows, R. (2012). Living with the H-Index? Metric assemblages in the contemporary academy. Sociological Review, 60, 355-372.

Court, S. e G. Kinman (2009). Tackling stress in higher education. London: University and College Union.

Crang, M. (2007). Flexible and fixed times working in the academy. Environment and Planning A, 39, 509-14.

De Angelis, M. e D. Harvie (2009). "Cognitive capitalism" and the rat race: How capital measures immaterial labour in British universities. Historical Materialism, 17, 3-30.

Deem, R., S. Hillyard e M. Reed (2007). Knowledge, higher education, and the new managerialism: The changing management of UK universities. Oxford: Oxford University Press.

Dyer-Witheford, N. (1999). Cyber-Marx: Cycles and circuits of struggle in high-technology capitalism. Urbana: University of Illinois Press.

Dyer-Witheford, N. (2005). Cognitive capitalism and the contested campus. In G. Cox e J. Krysa (a c. di) Engineering Culture: On the Author as (Digital) Producer, (pp. 71-93). New York: Autonomedia.

Gill, R. (2010). Breaking the silence: The hidden injuries of the neoliberal university. In R. Ryan-Flood e R. Gill (a c. di), Secrecy and Silence in the Research Process: Feminist Reflections, (pp.228-244). Abingdon: Routledge.

Gill, R. (2014). Academics, cultural workers and critical labour studies. Journal of Cultural Economy, 7: 12-30.

Giroux, H. (2002). Neoliberalism, corporate culture, and the promise of higher education: The university as a democratic public sphere. Harvard Educational Review, 72, 425-463.

Gorz, A. (2010). The immaterial: Knowledge, value and capital. London: Seagull Books.

Gray, D. (2004). Doing research in the real world. London: SAGE. 
Gulli, B. (2009). Knowledge production and the superexploitation of contingent academic labor. Workplace: A Journal for Academic Labor, 16, 1-30.

Hall, G. (2016). The Uberfication of the university. Minneapolis: University of Minnesota Press.

Higher Education Statistics Agency (2016). Data and Analysis. https://www.hesa. ac.uk/data-and-analysis.

Kinman, G. e S. Wray (2013). Higher stress: A survey of stress and well-being among staff in higher education. London: University and College Union.

Krippendorff, K. (2004). Content analysis: An introduction to its methodology. Thousand Oaks: SAGE

Lorenz, C. (2012). If you're so smart, why are you under surveillance? Universities, neoliberalism, and new public management. Critical Inquiry, 38, 599-629.

Lune, H. e B. Berg (2017). Qualitative research methods for the social sciences. Harlow: Pearson.

Miller, H. (1991). Academics and their labour process. In C. Smith, D. Knights e H. Willmott (a c. di) White-collar work: The non-manual labour process, (pp. 109-137). Basingstoke: Macmillan.

Norkus, M., C. Besio e N. Baur (2016). Effects of project-based research work on the career paths of young academics. Work Organisation, Labour \& Globalisation, 10, 9-26.

Peters, M. (2003). Classical political economy and the role of universities in the new knowledge economy. Globalisation, Societies and Education, 1, 153-168.

Pratt, L. R. (1997). Disposable faculty: Part-time exploitation as management strategy. In C. Nelson (a c. di). Will teach for food: Academic labor in crisis, (pp. 264-277). Minneapolis: University of Minnesota Press.

Prichard, C. e H. Willmott (1997). Just how managed is the McUniversity? Organization Studies, 18, 287-316.
Ross, A. (2000). The mental labor problem, Social Text, 18, 1-31.

Shumar, W. (1995). Higher education and the state: The irony of Fordism in American universities. In J. Smyth (a c. di), Academic work: The changing labour process in higher education, (pp. 84-98). Buckingham: Society for Research in Higher Education e Open University Press.

Slaughter, S. e L. Leslie (1999). Academic capitalism: Politics, policies, and the entrepreneurial university. Baltimore: Johns Hopkins University Press.

Tancred-Sheriff, P. (1985). Craft, hierarchy and bureaucracy: Modes of control of the academic labour process. Canadian Journal of Sociology, 10, 369-390.

Tirelli, V. (1999). Adjuncts and more adjuncts: Labor segmentation and the transformation of higher education. In R. Martin (a c. di), Chalk Lines: The Politics of Work in the Managed University, (pp. 181-201). Durham: Duke University Press.

University and College Union (2014). UCU survey of work-related stress. London: University and College Union.

University and College Union (2016). Workload is an education issue: UCU workload survey report 2016. London: University and College Union. https://www.ucu.org. uk/ media/8195/Workload-is-an-education-issue-UCU-workload-survey-report-2016/pdf/ ucu_workloadsurvey_fullreport_jun16.pdf

Watts, J. e N. Robertson (2011). Burnout in university teaching staff: A systematic literature review. Educational Research, 53, 33-50.

Willmott, H. (1995). Managing the academics: Commodification and control in the development of university education in the UK. Human Relations, 48, 993-1027.

Wright, S. (2002). Storming heaven: Class composition and struggle in Italian autonomist Marxism. London: Pluto. 
